\title{
Cenon-sur-Vienne - Rivière Clain
}

Jean-François Mariotti

URL : http://journals.openedition.org/adlfi/573

ISSN : 2114-0502

Éditeur

Ministère de la culture

Référence électronique

Jean-François Mariotti, « Cenon-sur-Vienne - Rivière Clain », ADLFI. Archéologie de la France -

Informations [En ligne], DRASSM (subaquatique terrestre), mis en ligne le 01 mars 2008, consulté le 19 avril 2019. URL : http://journals.openedition.org/adlfi/573

Ce document a été généré automatiquement le 19 avril 2019

(c) Ministère de la Culture et de la Communication, CNRS 


\title{
Cenon-sur-Vienne - Rivière Clain
}

\author{
Jean-François Mariotti
}

Date de l'opération : 2003 - 2008 (PI)

1 En 2007, un ensemble de blocs et trois chapiteaux antiques ont été mis au jour dans la rivière Clain au sud de Châtellerault (Fig. $\mathrm{n}^{\circ} 1$ : Chapiteau antique mis au jour à la confluence du Clain et de la Vienne). Situé au nord de la commune de Cenon-sur-Vienne, au lieu-dit Bretaigne, Fort-Clan, le site immergé dans le Clain est à $200 \mathrm{~m}$ en amont de la confluence avec la Vienne.

2 Au cours de prospections aériennes menées en 1989 sur la confluence de la Vienne et du Clain, Alain Ollivier, archéologue municipal de la ville de Poitiers, détecta un bâtiment dont l'emprise était marquée par l'arrachement des pierres lors des labours. Un sondage effectué cette même année sous sa direction (Ollivier, 1989) data cet ensemble des $\mathrm{III}^{\mathrm{e}} \mathrm{s}$. et IV ${ }^{\text {e }}$., sans toutefois identifier le bâtiment.

3 Ce sondage fournit aussi des indications intéressantes sur l'occupation de la confluence depuis la période protohistorique jusqu'à la période médiévale. Sur les indications d'Alain Ollivier, une plongée de reconnaissance eut lieu en 1996 dans le Clain ; elle permit le repérage des blocs immergés dans le chenal, à la hauteur du bâtiment. Une plongée de reconnaissance en 2006 et une prospection subaquatique engagée en 2007 par le service régional de l'Archéologie, précisèrent les observations faites dix ans auparavant. La topographie des vestiges immergés restitua deux alignements de pieux, un alignement de blocs grossièrement équarris. À ces traces d'aménagements s'ajoute un décaissement de berge dont la nature anthropique est fort probable.

4 La confluence de deux rivières constitue en général un emplacement stratégique. La protection naturelle, les ressources halieutiques, les possibilités de commerce et le contrôle de celui-ci, offerts par les deux cours d'eau, expliquent l'occupation précoce de ces territoires. Fort-Clan n'échappe pas à cette règle et les premières traces d'occupation remontent au Néolithique «avec une phase importante de développement au Bronze final» (Métais, 2001, p. 79). 
5 Les confluences réunissent aussi les conditions favorables à la fondation de ports. Dans ces contextes, les affluents de taille et de débit moindres étaient privilégiés pour installer les structures ou les aménagements de berge. Les vestiges mis au jour dans le Clain à FortClan semblent répondre à cette logique, au regard des caractéristiques des deux rivières.

6 De fait, la sécurité qu'offraient ces sites incitait des villes à y implanter leurs zones portuaires (Izarra, 1993). L'agglomération antique du Vieux-Poitiers (communes de Naintré et de Cenon-sur-Vienne) trouva certainement dans sa proximité avec la confluence, des facteurs déterminants pour sa naissance et son développement (Bourgeois, 2000, p. 165). La relation entre cette cité et le site de Fort-Clan

7 est probable (les vestiges d'un important établissement gallo-romain en rive gauche du Clain, dont la fouille est actuellement en cours, et ceux de la rive droite, témoignent d'une occupation jusqu'à l'extrême limite de la confluence).

8 La prospection-inventaire subaquatique de 2007 avait pour objectifs d'évaluer l'emprise du site, de topographier les vestiges et de prélever les chapiteaux.

9 Le Clain présente dans ce secteur un chenal d'une largeur de $30 \mathrm{~m}$ environ, et d'une profondeur moyenne de 2,40 $\mathrm{m}$. L'alignement de blocs, entrevu dès 1996 , se trouve à une cinquantaine de mètres en amont de la confluence. Placé à $8 \mathrm{~m}$ de l'actuelle berge, il semble se développer dans l'axe d'une trace de bâti observable en bord de berge.

10 La topographie effectuée sur ces blocs est incomplète : seule la partie centrale plus visible a été relevée. Longue de $13 \mathrm{~m}$ et large de $1,70 \mathrm{~m}$, son emprise est de $22 \mathrm{~m}^{2}$. Les blocs topographiés, au nombre de vingt-trois, forment la couche supérieure de cet aménagement. Un sondage a permis d'atteindre le niveau inférieur de l'assise: deux couches de blocs composent cette structure, lui conférant une élévation moyenne de $1 \mathrm{~m}$.

11 Les blocs qui composent cet ensemble sont grossièrement taillés, d'un module sensiblement homogène, ils ont une longueur moyenne de 1,50 m pour une largeur et une épaisseur moyenne de 0,50 $\mathrm{m}$. Des prélèvements pour une caractérisation géologique seraient nécessaires pour en déterminer l'origine.

12 L'agencement des blocs peut correspondre à un quai, son développement parallèle à la berge exclut toute autre possibilité, hormis un bief de moulin. L'utilisation de blocs mal équarris dans un appareillage grossier contraste avec l'autre aménagement de berge connu à ce jour sur le Clain et fouillé en 1991 (culées de pont en appareillage soigné sur un radier de pieux en chêne, ensemble daté des $\mathrm{I}^{\mathrm{er}}$ s.-II ${ }^{\mathrm{e}}$ s. (Grandjean, 1991). Le démontage d'une structure plus ancienne, implantée sur la confluence et le réemploi des blocs pour l'aménagement d'un quai est une possibilité.

13 L'environnement des chapiteaux C1, C2 et C3 est identique. Placés en contrebas de l'aménagement, ils se trouvent à une profondeur moyenne de 2,60 $\mathrm{m}$. Cette partie du chenal présente une concentration hétérogène de blocs de modules variables. Le sédiment qui les lie est compact, formé de sable, de gravier et de cailloux de petite taille.

14 Les parties hors sédiment des blocs et des chapiteaux ont une surface indurée. On retrouve de nombreux fragments de roche calcaire feuilletée dans le lit du Clain, correspondant certainement à la période du Kimméridgien ; ou affleurant parfois, dans les parties érodées du chenal en grandes dalles notamment à l'extrémité de la confluence.

15 Le sondage entrepris au pied des blocs a d'autre part révélé deux alignements de pieux (douze pieux d'un diamètre moyen de $8 \mathrm{~cm}$ à $10 \mathrm{~cm}$ ), parallèles entre eux, formant un 
angle de $45^{\circ}$ avec les blocs. Une céramique a aussi été trouvée: il s'agit d'un pot globulaire à petit empiétement et épaulement haut. La lèvre est triangulaire, débordante et déversante. La pâte à cuisson réductrice présente une surface rugueuse avec de grosses inclusions visibles à sa surface. Cette céramique peut être placée dans une fourchette chronologique qui irait de l'Antiquité tardive à la période mérovingienne.

Neuf autres pièces métalliques, dont deux fers de haches, ont été mises au jour à la faveur des décapages entrepris pour le prélèvement des chapiteaux $\mathrm{C} 2$ et $\mathrm{C} 3$. Ces haches présentent un dos droit ou légèrement convexe, un tranchant asymétrique et un emmanchement parallèle au talon. Cette forme les rattache au groupe III, $\mathrm{K}$ de la typologie établie par Wolfgang Hüberner (1980). Patrick Périn, dans son ouvrage La datation des tombes mérovingiennes, définit quand à lui quatre groupes en fonction de la forme du dos, de l'angle formé par l'axe de l'emmanchement et le dos et du développement du tranchant (Périn, 1980, p. 226). Les deux exemplaires de Cenon entrent sans conteste dans la catégorie $4 \mathrm{~B}$ (tranchant asymétrique). Une étude du mobilier funéraire mérovingien (Legoux et al., 2006, p. 54) les placerait aux VI ${ }^{\mathrm{e}} \mathrm{s} . \mathrm{VII}{ }^{\mathrm{e}} \mathrm{s}$.

Le site de Fort-Clan a connu une occupation précoce. À la période gallo-romaine, l'activité humaine y fût certainement importante, les vestiges de bâtiments sur les rives gauche et droite du Clain en témoignent.

L'utilisation avérée des cours d'eau mineurs comme le Clain, pour le transport de marchandises aux périodes antiques, la proximité de l'agglomération du Vieux-Poitiers et les avantages offerts par les sites de confluence, rendent probable une activité fluviale à cet endroit. L'alignement de blocs mis au jour est indéniablement une structure ou un aménagement de berge dont la finalité est inconnue. Sa datation, en l'absence d'éléments constitutifs en bois, est difficile. Son agencement sommaire et les matériaux grossiers qui le composent, ne l'apparentent guère aux ouvrages gallo-romains découverts en milieu fluvial et étudiés. Les trois chapiteaux (probablement de l'Antiquité tardive) trouvés près des blocs, ainsi que les nombreux fragments de tegula ne peuvent leur être actuellement associés. L'origine de ces autres vestiges est probablement le bâtiment gallo-romain de la confluence ou de la rive gauche du Clain dont la fouille est en cours. La découverte de mobilier métallique de la période mérovingienne dans un niveau inférieur laisse supposer que les bâtiments devaient être encore visibles au haut Moyen Âge. On peut toutefois s'étonner de l'abandon manifeste des chapiteaux: leur facture soignée, le marbre employé, le réemploi fréquent dans les premiers édifices chrétiens d'éléments d'architecture antique, leur conférait une valeur marchande certaine. La faible profondeur du Clain et le poids relatif de ces chapiteaux invalident, d'autre part, le scénario d'une perte de chargement; leur récupération étant possible même à cette époque.

\section{ANNEXES}


Fig. $n^{\circ} 1$ : Chapiteau antique mis au jour à la confluence du Clain et de la Vienne

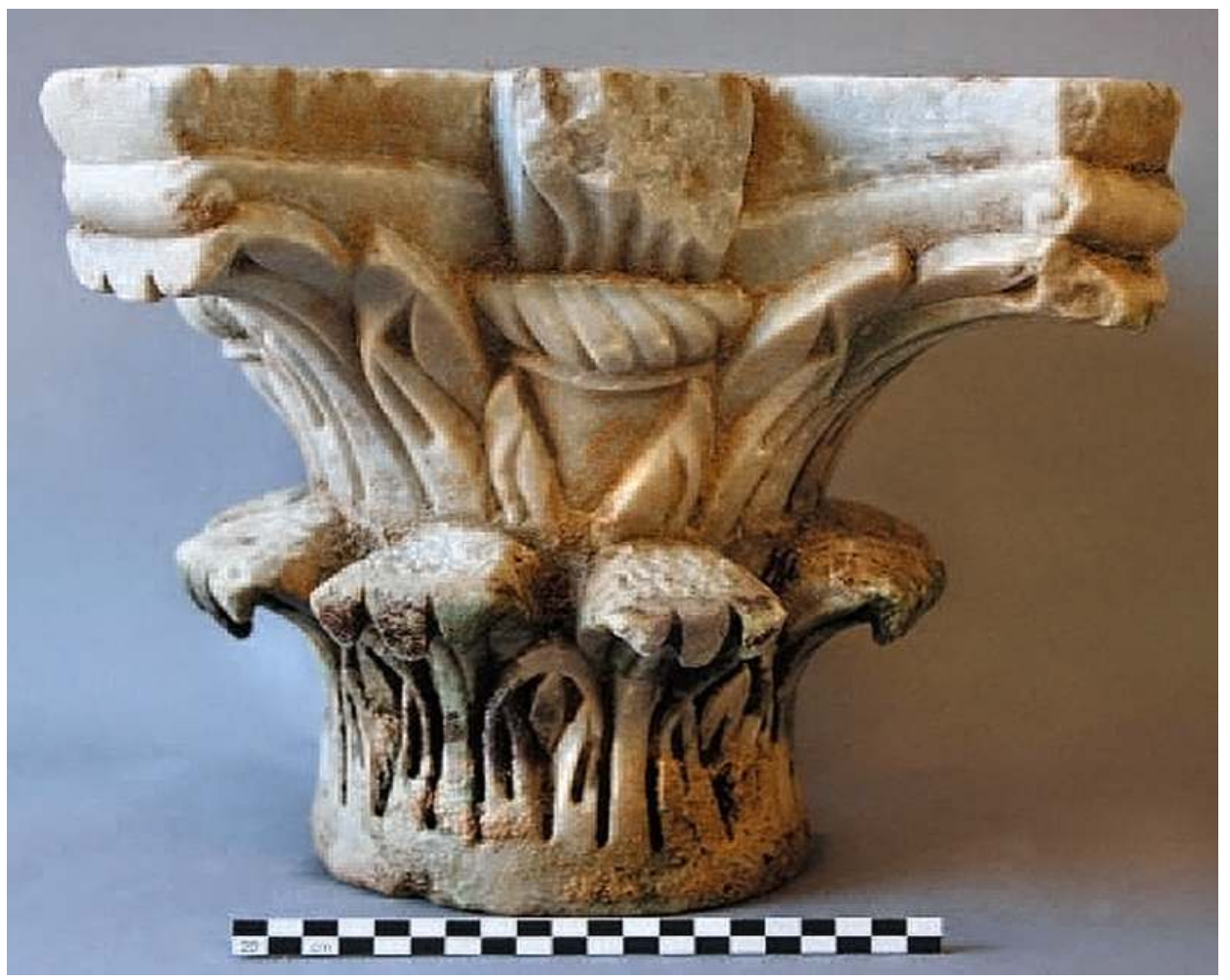

Auteur(s) : Mariotti, Jean-François (SRA). Crédits : Mariotti, Jean-François (2008)

\section{INDEX}

operation Prospection inventaire (PI)

Index géographique : Poitou-Charentes, Vienne (86), Cenon-sur-Vienne

Index chronologique : Antiquité tardive, Bas-Empire, haut Moyen Âge, Protohistoire

Thèmes : activités commerciales, aménagement de berge, céramique mérovingienne, chapiteau, chenal, commerce fluvial, édifice, fleuve, hache à talon, pieu, port, quai, rivière, topographie, transport sur eau

\section{AUTEURS}

\section{JEAN-FRANÇOIS MARIOTTI}

